\title{
Development and justification of the parameters of the coulter group of grain drills
}

\author{
Viktor Kurushin ${ }^{1, *}$, Vladimir Kurdyumov ${ }^{1}$ and Ivan Sharonov ${ }^{1}$ \\ ${ }^{1}$ Ulyanovsk State Agricultural University named after P. A. Stolypin, Ulyanovsk, RUSSIA
}

\begin{abstract}
The urgency of creation and use of resource-saving means of mechanization of agriculture is proved. Proposed grain drill, performing the sowing of grain crops on stubble background. Its use allows to sow seeds in the soil with minimum costs due to the reduction of the number of technological operations. The theoretical substantiation of the grain seeder, on the basis of which the optimal design and operating parameters are revealed, is carried out.
\end{abstract}

In the cultivation of crops plays a key role sowing, which is carried out in various ways. Currently, in Russia, most of the agricultural land is sown by tra-ditional technology, which includes about ten technological methods. Due to the large number of operations, the time of sowing increases, which does not always allow to meet the specified agro-technical deadlines, undesirable soil compaction occurs, erosion processes of the soil increase. In total, all this leads to a decrease in the yield and a significant increase in the cost of production, as a result of which agricultural production becomes unprofitable. Therefore, in recent years, more and more attention is paid to the sowing of zero technology. Many scien-tists have recognized this technology as cost-effective and advanced from an en-vironmental point of view.

However, for the implementation of seeding on this technology, such means of mechanization are necessary that would sow the seed material in ac-cordance with agrotechnical requirements.

Based on the analysis of existing means of mechanization of sowing of grain crops, we have developed a grain seeder (fig. 1), which carries out sowing without pre-tillage, while loosening the top layer of soil and introducing plant residues into it, which eventually eliminates the introduction of mineral fertilizers.

The quality of operation of the proposed grain seeder $[1,2,3,4]$ depends on the correctness of the optimization of design-mode and technological parameters. Since we use spherical and flat discs as coulters, it is necessary to determine their parameters, which will comply with agricultural requirements.

Find the diameter of the disk [5] from the condition of providing a given depth of tillage (fig. 2). It should be borne in mind that the working bodies have individual placement on the frame of the seeder.

\footnotetext{
${ }^{*}$ Corresponding author: kurushin.viktor@yandex.ru
} 


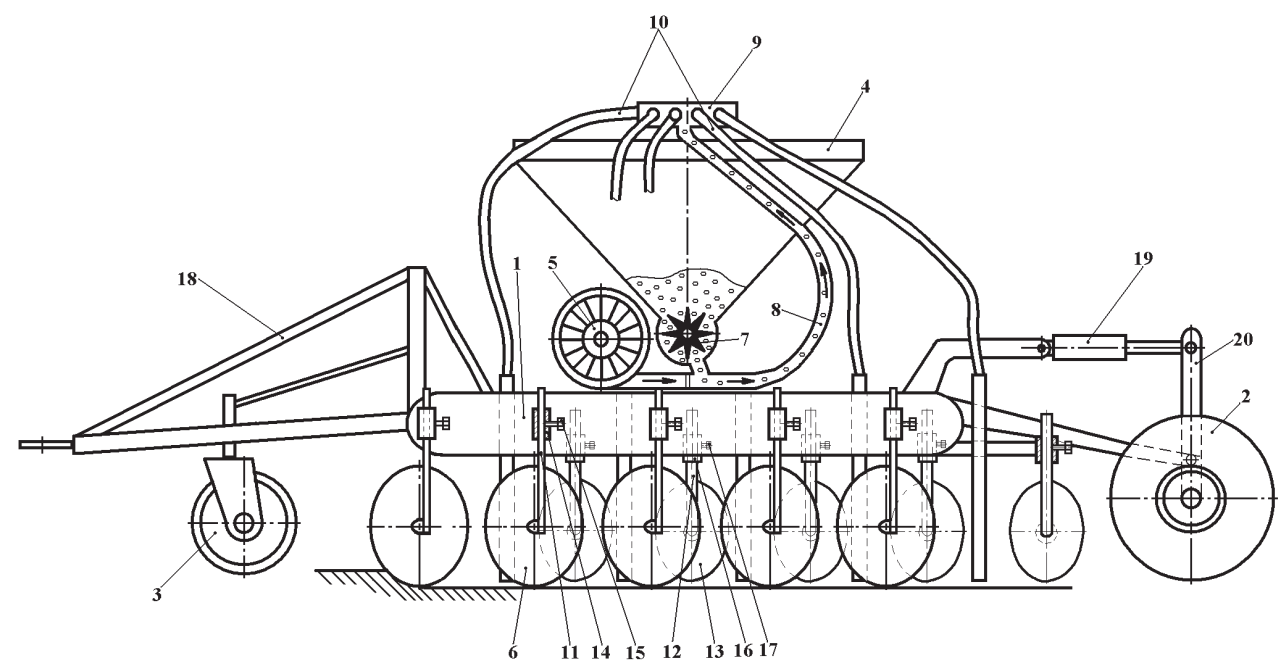

1 - frame; 2,3 - support and running wheels; 4 - hopper; 5 - fan; 6 - coulters; 7 - coil sowing machine; 8 - the vas deferens; 9 - dividing head; 10 - seed pipes; 11 - rotary racks; 12 - flat disc racks; 13 - flat discs; 14 - bracket; 15,17 - bolt; 16 - guide sleeve; 18 - coupling device; 19 - hydraulic cylinder, 20 supporting mechanism

Fig. 1. Scheme of the proposed seeder.

Determine the diameter of the spherical disk, taking into account the angle of its attack:

$$
D=\frac{b^{2}}{4 a \sin ^{2} \theta}+a
$$

where $\theta$ - the angle of attack of a spherical disk, hail.

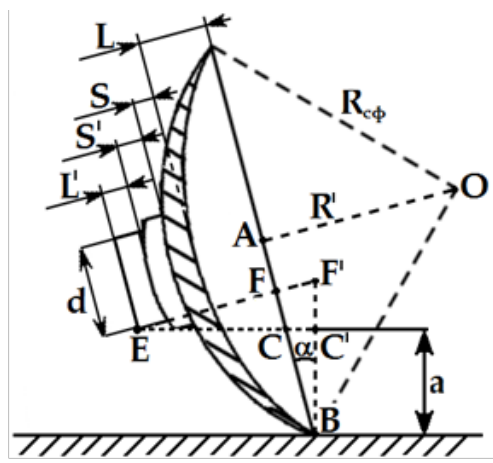

$\mathrm{a}$ - the depth of the disc in the soil; $\mathrm{d}$ - the diameter of the bearing housing; $\mathrm{L} / \mathrm{-}$ the thickness of the bearing housing; $\mathrm{S}$ - the thickness of the disc; $\mathrm{S} /$ - the thick-ness of the flange axis of the disc; $\mathrm{L}$ - the boom of the deflection disk

Fig. 2. To determine the diameter of the disc.

Knowing the diameter, we find the angle of attack of the spherical disk:

$$
\sin ^{2} \theta=\frac{b^{2} \cos \alpha}{4 a\left(D-\frac{a}{\cos \alpha}\right)},
$$




$$
\theta=\arcsin \frac{b \sqrt{\cos \alpha}}{2 \sqrt{a\left(D-\frac{a}{\cos \alpha}\right)}}
$$

where $\alpha$ - angle of inclination of the spherical disk, hail.

So as disk use without the tilt, that is $\alpha=0$. In this case:

$$
\theta=\arcsin \frac{b}{2 \sqrt{a(D-a)}}
$$

Thus, the angle of attack of a spherical disk depends on the width and depth of the furrow formed in the soil, as well as the diameter of the disk.

Let us consider the case of displacement of the "compressible" layer, as for the real layer occupying an intermediate position between the "compressible" and "incompressible" the same laws are preserved.

Let's take the point of the formation descent from the disk as the beginning of a fixed coordinate system.

The trajectory of the particles is a parabola and obeys the laws of motion for the body thrown at an angle to the horizon.

Consider the trajectory of the soil particle (fig. 3).

$$
\begin{gathered}
x=v_{0} \cos ^{2} \lambda\left(1+\operatorname{tg} \varphi_{0} \operatorname{tg} \lambda\left(\frac{\cos \lambda_{0}-\cos \lambda \cos \psi}{\sin \lambda \sin \psi}\right)\right) t \\
y=v_{0} \cos \lambda \cos \gamma\left(1-\operatorname{tg} \varphi_{0} \operatorname{ctg} \lambda\left(\frac{\cos \psi-\frac{\cos \gamma_{0}}{\cos \gamma}}{\sin \psi \operatorname{ctg} \gamma}\right)\right) \\
z=v_{0} \cos \lambda \cos \sigma\left(1-\operatorname{tg} \varphi_{0} \operatorname{ctg} \lambda\left(\frac{\cos \psi-\frac{\cos \sigma_{0}}{\cos \sigma}}{\sin \psi \operatorname{ctg} \lambda}\right)\right) t-\frac{g t^{2}}{2},
\end{gathered}
$$

where $\varphi_{0}$ - the angle of friction of the soil on the surface of the disc, hail; $\lambda$ - the angle between the inner normal $O N$ to the working surface and the axis $O X$, hail; $\gamma$ - the angle between the inner normal $O N$ to the work surface and the axis $O Y$, hail; $\sigma$ - the angle between the inner normal $O N$ to the work surface and the axis $O Z$, hail; $\psi$ - the angle between the normal and the speed of this point of the working surface of the disk; $\lambda_{0}, \gamma_{0}, \sigma_{0}, \psi_{0}$ - length of sides of spherical triangles, m; $\cos \lambda_{g}, \cos \gamma_{g}, \cos \sigma_{g}$ - guiding vectors of speed of any point of the working surface of a rotating disk 


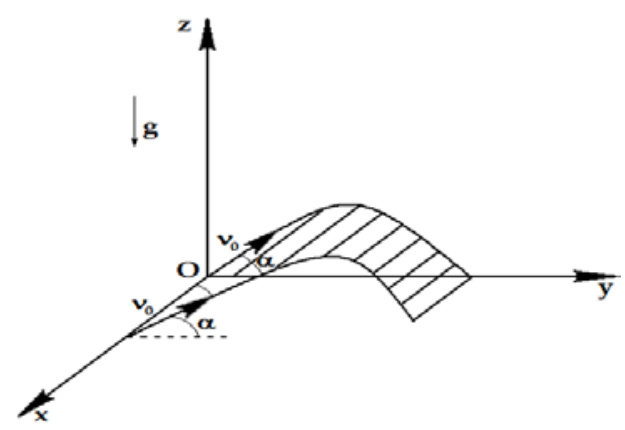

Fig. 3. Trajectory of soil particles.

With the help of formulas $(5-7)$ it is possible to determine:

- maximum lifting height of soil particles $H_{\max }$;

- range of movement of soil particles $L_{\Pi}$.

To do this, calculate the rise time $t_{\text {pod }}$ and travel time $t_{p o l}$ soil particles.

$$
\begin{aligned}
& t_{p o l}=\cos \lambda \cos \sigma\left(1-\operatorname{tg} \varphi_{0} \operatorname{ctg} \lambda\left(\frac{\cos \psi-\frac{\cos \sigma_{0}}{\cos \sigma}}{\sin \psi \operatorname{ctg} \lambda}\right)\right) v_{0} \\
& t_{p o l}=2 \cos \lambda \cos \sigma\left(1-\operatorname{tg} \varphi_{0} \operatorname{ctg} \lambda\left(\frac{\cos \psi-\frac{\cos \sigma_{0}}{\cos \sigma}}{\sin \psi \operatorname{ctg} \lambda}\right)\right) v_{0} / g \\
& H_{\max }=\left(\cos \lambda \cos \sigma\left(1-\operatorname{tg} \varphi_{0} \operatorname{ctg} \lambda\left(\frac{\cos \psi-\frac{\cos \sigma_{0}}{\cos \sigma}}{\sin \psi \operatorname{ctg} \lambda}\right)\right) v_{0}\right)^{2} / g ; \\
& L_{p}=y(t)=\cos \lambda \cos \gamma\left(1-\operatorname{tg} \varphi_{0} \operatorname{ctg} \lambda\left(\frac{\cos \psi-\frac{\cos \gamma_{0}}{\cos \gamma}}{\sin \psi \operatorname{ctg} \gamma}\right)\right) v_{0} t_{p o l}= \\
& =2 \cos \lambda \cos \sigma\left(1-\operatorname{tg} \varphi_{0} \operatorname{ctg} \lambda\left(\frac{\cos \psi-\frac{\cos \sigma_{0}}{\cos \sigma}}{\sin \psi \operatorname{ctg} \lambda}\right)\right) v_{0}^{2} / g .
\end{aligned}
$$

Consequently, the range of movement and the maximum height of the soil particle rise depends on the speed of the unit and the physical and mechanical properties of the soil.

Next, we determine the diameter of the levelling disc, the main purpose of which is to fill the groove obtained from the spherical disc to form a smooth surface, i.e. it must smooth the 
previously obtained soil hillock. The width of this ridge represents the range of movement of soil particles (fig. 4).

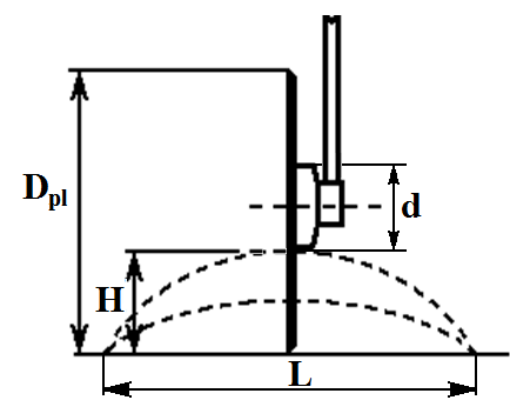

Fig. 4. To determine the diameter of a flat disc.

$$
D_{p l}=\frac{L_{b}^{2}}{4 H \sin ^{2} \theta^{\prime}}+H,
$$

where $L_{\mathrm{b}}$ - the width of the resulting tubercle, m; $\theta^{\prime}$ - the angle of attack of a flat disk, hail.

If you imagine $D_{p l}$ through construction parameter, then

$$
D_{p l}=2\left(H+\frac{d}{2}\right) .
$$

Using the analogy of flat and spherical disks, we obtain an expression for determining the diameter of a flat disk taking into account its design features. (fig. 5).

We construct a scheme to determine the distance between the flat and spherical disk

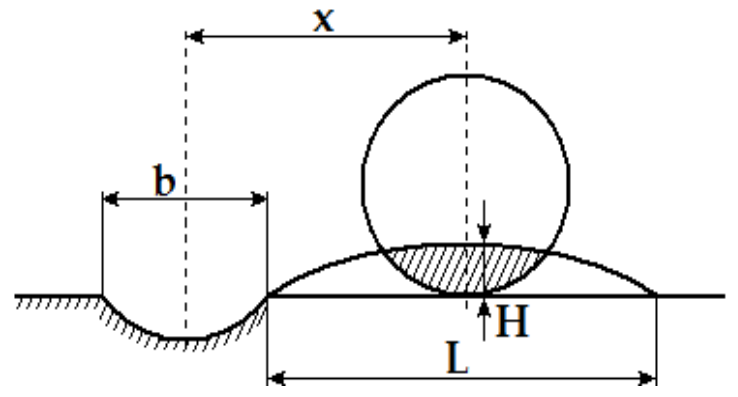

Fig. 5. To determine the distance between disks.

Figure 5 shows that the distance between flat and spherical disks:

$$
x=\frac{b}{2}+\frac{L_{n}}{2}=\frac{b+L_{n}}{2},
$$

where $b$ - the width of the cavity formed after the passage of the spherical disk, $\mathrm{m}$.

Based on the above, we find the required diameter of the flat disk: 


$$
D_{p l}-H=\frac{L_{p}^{2}}{4 H \sin ^{2} \theta}
$$

and, knowing this diameter, determine the angle of attack:

$$
\theta^{\prime}=\arcsin \frac{L_{p}}{2 \sqrt{H\left(D_{p l}-H\right)}} .
$$

Consequently, the angle of attack of the leveling flat disk depends on the distance of movement of the soil particle, the diameter of the disk, as well as the height of the soil tubercle formed by the spherical disk.

A flat disc is installed to obtain a perfect alignment after the passage of the seeder. Therefore, to meet this condition, it is necessary to determine how much soil the spherical disc throws.

After a series of transformations, we obtain the formula:

$$
V=\frac{S \cos \lambda \cos \sigma\left(1-\operatorname{tg} \varphi_{0} \operatorname{ctg} \lambda\left(\frac{\cos \psi-\frac{\cos \sigma_{0}}{\cos \sigma}}{\sin \psi \operatorname{ctg} \lambda}\right)\right) L^{2}}{2 \cos \lambda \cos \gamma\left(1-\operatorname{tg} \varphi_{0} \operatorname{ctg} \lambda\left(\frac{\cos \psi-\frac{\cos \gamma_{0}}{\cos \gamma}}{\sin \psi \operatorname{ctg} \gamma}\right)\right)}-
$$$$
\frac{S g L_{b}{ }^{3}}{6 \cos \lambda \cos \gamma\left(1-\operatorname{tg} \varphi_{0} \operatorname{ctg} \lambda\left(\frac{\cos \psi-\frac{\cos \gamma_{0}}{\cos \gamma}}{\sin \psi \operatorname{ctg} \gamma}\right)\right)^{2} v^{2}}
$$

where $S$ - length of the formed hillock of soil, m; $L_{b}$ - the width of the formed tu-bercle of the soil, $\mathrm{m}$.

From the obtained formula it follows that the volume of soil depends on the speed of the unit, the size of the formed hillock of the soil and the physical and mechanical properties of the soil.

On the basis of theoretical studies, a formula for determining the relation-ship of the angles of attack of spherical and flat discs is obtained:

$$
\theta=\arcsin b \sin \theta^{\prime} \sqrt{\frac{H}{a\left(L^{2}+(a-H)\right) 4 H \sin ^{2} \theta^{\prime}}} .
$$

Using the expression (17), it is possible to find the optimal parameters of a pair of disks, taking into account the technological requirements for the process of grain sowing. The angle of attack of a spherical disk is optimal $\theta=10$ hail, flat $\theta^{\prime}=23$ hail. The results were confirmed in laboratory studies. 


\section{References}

1. V. I. Kurdyumov, E. S. Zykin, V.V. Kurushin, Seeder, Patent No. 90961 Russian Federation, publ. 27.10.2010, byul. No. 3.

2. V. I. Kurdyumov, E. S. Zykin, V.V. Kurushin, Seeder, Patent No. 90962 Russian Federation, publ. 27.10.2010, byul. No. 3 .

3. N. E. Rudenko, K. D. Padalin, How to reduce energy costs and improve the quality indicators under continuous tillage, Vestnik APK Stavropol, 2014, № 1(13), pp. 66-68.

4. N. P. Laryushin, A. V. Zhukov, Relevance of resource-saving technology of grain crops, Modern science-intensive technologies, 2009, № 6, pp. 18-20.

5. V. S. Nestyak, K. T. Mambetalin, Tillage when direct seeding, Bulletin of Altai state agrarian University, 2011, № 12(86), pp. 99-103. 